\title{
Honduras: Promover nuevos servicios de salud reproductiva es costo-efectivo
}

Frontiers in Reproductive Health

Follow this and additional works at: https://knowledgecommons.popcouncil.org/departments_sbsr-rh

Part of the Demography, Population, and Ecology Commons, International Public Health Commons, Maternal, Child Health and Neonatal Nursing Commons, and the Public Health Education and Promotion Commons How does access to this work benefit you? Let us know!

\section{Recommended Citation}

"Honduras: Promover nuevos servicios de salud reproductiva es costo-efectivo," FRONTERAS Resumen de Investigación Operative. Ciudad de México: Population Council, 2001. 


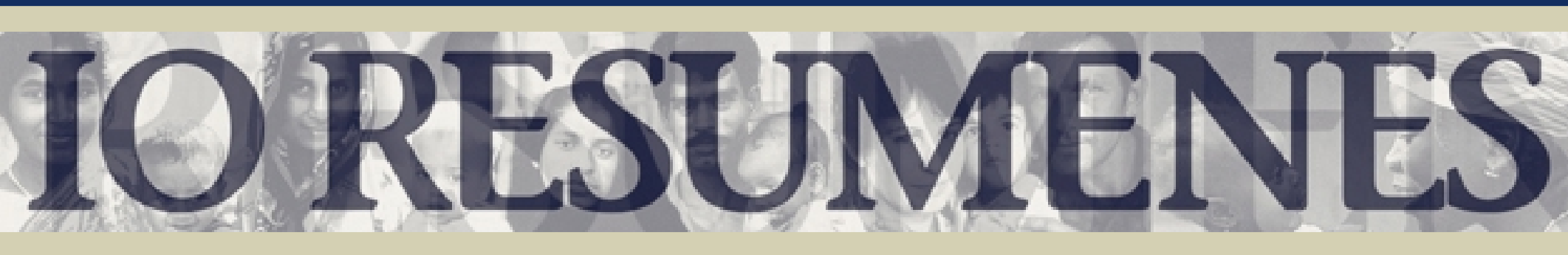

\section{Honduras \\ Acceso a servicios \\ Promover nuevos servicios de salud reproductiva es costo-efectivo}

\section{Investigación Operativa}

Resumen 18

\begin{abstract}
Seis centros de salud rurales en Honduras impartieron pláticas de diez minutos y usaron folletos de bajo costo para anunciar la disponibilidad de los servicios de inserción de DIU, del inyectable DMPA y del Papanicolaou, con lo cual, a un costo razonable, el uso de estos servicios aumentó. Este tipo de promoción se hará en otras clínicas donde las auxiliares de enfermería hayan sido capacitadas para prestar estos servicios.
\end{abstract}

\section{Antecedentes}

En 1999, la Secretaría de Salud de Honduras (SSH) modificó las Normas de Atención Integral a la Mujer a fin de autorizar a las auxiliares de enfermería a prestar los servicios de inserción de DIU, aplicación del inyectable DMPA y toma de Papanicolaou. Este cambio en las normas aborda el problema de la baja prevalencia en el uso de anticonceptivos en áreas rurales $(40 \%$, en contraste con $67 \%$ en áreas urbanas). El cambio también refleja los hallazgos de un estudio anterior, realizado por el Population Council, que mostró que las auxiliares de enfermería pueden proporcionar estos servicios de manera segura y con buena calidad. Anteriormente, las mujeres en las áreas rurales tenían un acceso limitado a los métodos de planificación familiar de larga duración, pues en los Centros de Salud Rurales (CESARES), a donde generalmente acuden, las proveedoras de salud son auxiliares de enfermería.

En el año 2000, el Population Council y la SSH evaluaron la eficacia y el costo de usar un sencillo folleto, distribuido por las auxiliares de enfermería, para promover el uso de los nuevos servicios. Doce CESARES (seis en el grupo de control y seis en el experimental) se eligieron al azar entre los 180 centros en los que las auxiliares de enfermería habían sido capacitadas para prestar los nuevos servicios. Las auxiliares de los centros del grupo experimental recibieron 500 folletos para distribuir entre la población, dieron pláticas de 10 minutos todos los días sobre los nuevos servicios y le pidieron a cada mujer que acudía a la clínica que distribuyera cinco folletos entre sus amigas y vecinas. Para evaluar la eficacia de la promoción, se comparó la asistencia a los nuevos servicios (Papanicolaou; nuevas usuarias y seguimiento a usuarias de DIU, y DMPA) durante tres meses antes y tres meses después de la intervención.

\section{Hallazgos}

- Las visitas para recibir los nuevos servicios aumentaron 22 por ciento en las clínicas del grupo experimental, comparado con el 12 por ciento de aumento en las clínicas del grupo de control. En el grupo experimental, las clínicas proporcionaron en promedio 7.7 servicios adicionales al mes, mientras que las clínicas del grupo de control proporcionaron sólo 0.9 servicios adicionales al mes, en promedio.

- Durante los tres meses posteriores a la intervención, las inserciones de DIU en las clínicas del grupo experimental aumentaron 50 por ciento, mientras que en las clínicas del grupo de control, disminuyeron 42 por ciento (ver la figura en la siguiente página). La aplicación del inyectable DMPA aumentó 36 por ciento en las clínicas del grupo experimental, contra un 19 por ciento en el grupo de control. En las clínicas del grupo experimental, las proveedoras tomaron 22 por ciento más pruebas de Papanicolaou, mientras que este servicio disminuyó 4 por ciento en el grupo de control. 

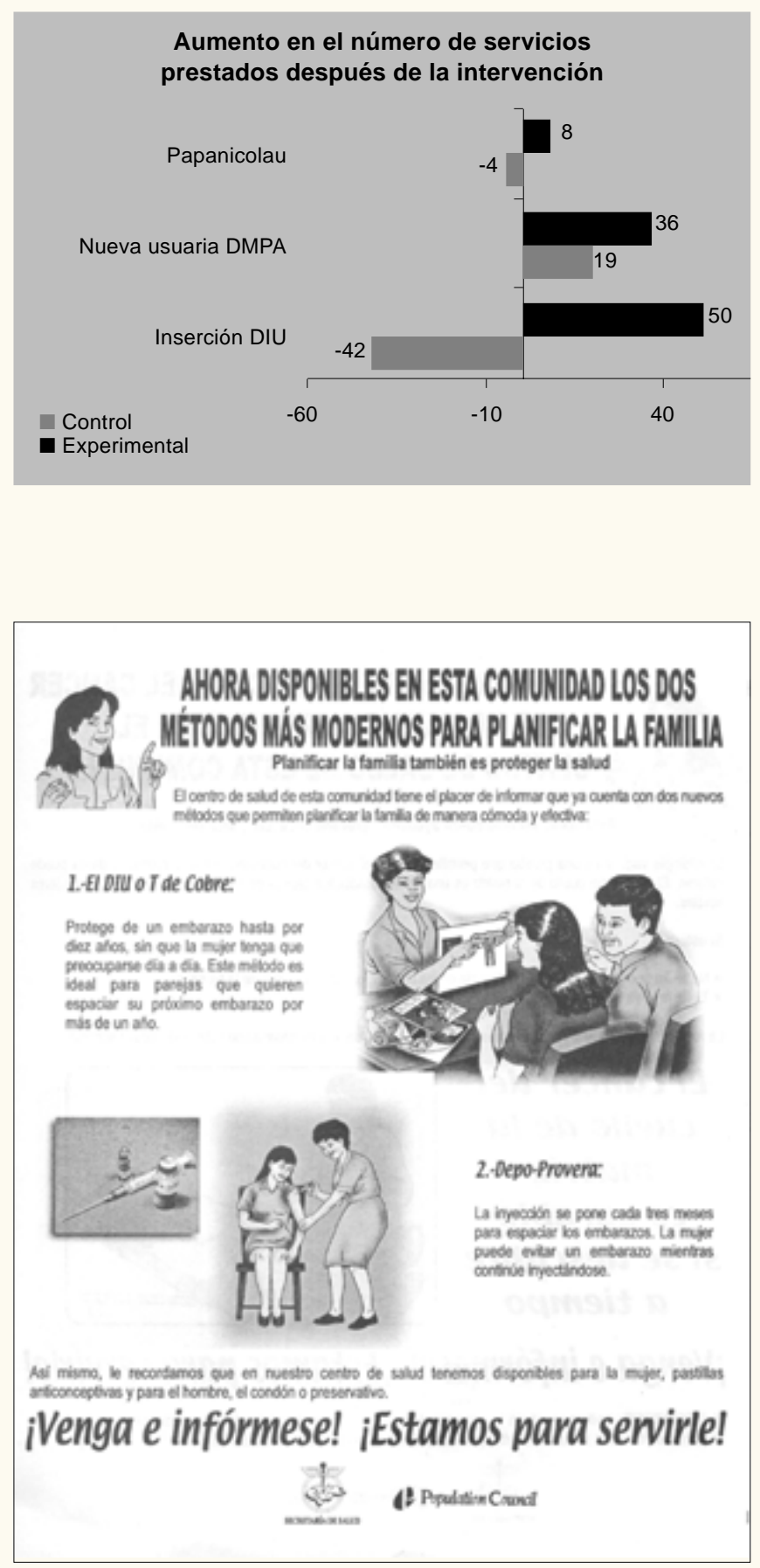

- Un análisis de costos encontró que el costo total de la intervención, incluyendo la capacitación, la supervisión y la producción de 25,000 folletos, fue de US\$950.

- Si consideramos el aumento en los servicios en estos tres meses en las seis unidades del grupo experimental, el costo por servicio adicional fue de US\$7.80. Extrapolando el efecto a seis meses y 100 unidades, equivaldría a 4,080 usuarias adicionales, y representaría un costo por usuaria de US\$0.13 - un costo razonable para este ámbito.

\section{Implicaciones normativas}

Esta simple estrategia de promoción dio como resultado un aumento en la demanda de los tres nuevos servicios de salud reproductiva, en especial de la inserción de DIU y de las inyecciones de DMPA. La intervención es eficaz en función del costo y se recomienda implementarla en todos los centros de salud rurales en donde se han instaurado los nuevos servicios.

\section{Utilización}

Basándose en los resultados de este estudio, la SSH planea proveer el folleto a todas las auxiliares de enfermería que han recibido capacitación para proveer los servicio, así como a las ONG que también proporcionan estos servicios.

\section{Fuente:}

Villanueva, Yadira, Lourdes Hernández, Irma Mendoza y Rebecka Lundgren. 1998. "Expansion of the role of nurse auxiliaries in provision of family planning services and cytology samples [Expansión del rol de las auxiliaries de enfermería en la oferta de servicios de planificación familiar y toma de citologías vaginales]," INOPAL Final Report. Washington, DC: Population Council. Disponible por correo electrónico: frontiers@pcdc.org

Este proyecto se llevó a cabo con el apoyo de la AGENCIA DE LOS ESTADOS UNIDOS PARA EL DESARROLLO INTERNACIONAL (USAID) bajo el Contrato No. CCP-95-00-00007-00. 